\title{
User Identification: Bio-Inspired Visual User Detection
}

\author{
W. Han* and I. S. Han** \\ Korea Advanced Institute of Science and Technology
}

\begin{abstract}
This paper describes the bio-inspired visual detection for the smart user experience based on neuromorphic system, mimicking the visual cortex. The neuromorphic system is investigated for visual detection of users and their reaction, based on the VLSI neurons and synapses by CMOS conductance and Hodgkin-Huxley formalism. The proposed neuromorphic system exhibits the biologically plausible intelligent function mimicking the cat's visual cortex experimentation of Hubel and Wiesel. The feasibility of bio-inspired vision is evaluated by visual detection of human head or pose under the limited natural environment. The visual detection in various environments of on-vehicle, glossy indoors, or dark conference room demonstrates as smart user interface to extend the internet service to IPTV as the smart phone of high user acceptance smart phone, with the robust function applicable to enhance the secure operation.
\end{abstract}

\section{Introduction}

The ubiquitous information technology or ambient aware service has been widely developed recently, and the convergence of information technology introduced the new paradigm of user experience like smart phone. The promotion of IPTV service to the market becomes an opportunity of telecom operators trying to offset decline in the traditional revenue with new enabling technology of smart TV. The flexible and intelligent visual user detection can transfer the successful factor of smart phone's user experience to the emerging smart IPTV.

There have been many works proposed recently for the robust and intelligent visual information processing with neuromorphic circuits and system, by mimicking both the functional and physiological characteristics of biological systems. We describe here the bio-inspired implementation of primary visual cortex, based on the Hodgkin-Huxley formalism and the experimentation of Hubel and Wiesel. The feasibility of neuromorphic implementation is investigated by simulation and experimentation based on CMOS technology, with demonstrated vision applications of face or pose detection in the robust environment. In this paper, we propose the new way of implementing the neuromorphic system for the primary visual cortex, inspired by the ideas on the primary visual cortex by Hubel and Wiesel's experimentation and the neurophysiological model of neuron by Hodgkin and Huxley. The design motivation is from the wellknown experimentation of simple cell in Figure 1 by Hubel and Wiesel.

The robust and intelligent visual processing of neuromorphic system can be used in a seamless and easy way to identify users of their age, peculiar reaction behavior to certain situations, which enables users through smart interface design letting their machine become a intelligent gateway to the internet.

\section{Neuromorphic system of visual cortex}

The physiological studies about visual cortex from the investigation of cat's striate cortex by Hubel and Wiesel have confirmed the consensus of knowledge, though there are many models about visual cortex. The idea on the primary visual cortex of simple cell motivated various theories of object recognition from characters to complex natural images. The controlled conductance by CMOS transistors is used as an element of our neuromorphic system, which have been studied for biologically plausible analogue-mixed neural networks VLSI.

The Hodgkin-Huxley $(\mathrm{H}-\mathrm{H})$ formalism is a widely adopted idea of neuron's biophysical characterisation and dynamics. The $\mathrm{H}-\mathrm{H}$ formalism is widely of interest for its biophysical dynamics, though its complexity in computation is prohibitively high. Hence asynchronous dynamics of the $\mathrm{H}-\mathrm{H}$ formalism is adopted as the idea of neuron model. Our circuit of CMOS voltage controlled-conductance has been investigated for various neural networks applications, from implementing synapses to neuron [6]. An electrical equivalent circuit model of Figure. 1 (a) is known as the empirical model of $\mathrm{H}-\mathrm{H}$ formalism, which describes quantitatively the dynamics of the voltage-dependent conductance. Although most of particular neural networks tasks do not exhibit any major advantages based on $\mathrm{H}-\mathrm{H}$ formalism, asynchronous spikes are considered as a principle element of high level or large scale neural computing system. The $\mathrm{H}-\mathrm{H}$ formalism is widely of interest for its biophysical dynamics, though its complexity in computation is prohibitively high. 
Hence asynchronous dynamics of the $\mathrm{H}-\mathrm{H}$ formalism is adopted as the idea of neuron model.
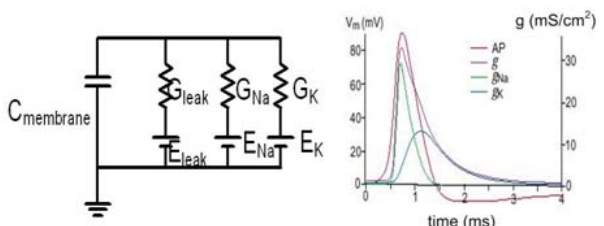

Figure 1. (a) An electrical equivalent circuit of a neuron, Hodgkin-Huxley formalism (b) dynamics of asynchronous spike and refractory period vs. the membrane potential [3]

The overall dynamic behaviour of biological neuron is illustrated by the ion-based conductance controlled by membrane potential (or Action Potential), with the illustrated dynamics of conductance in Figure 2(b) [4].

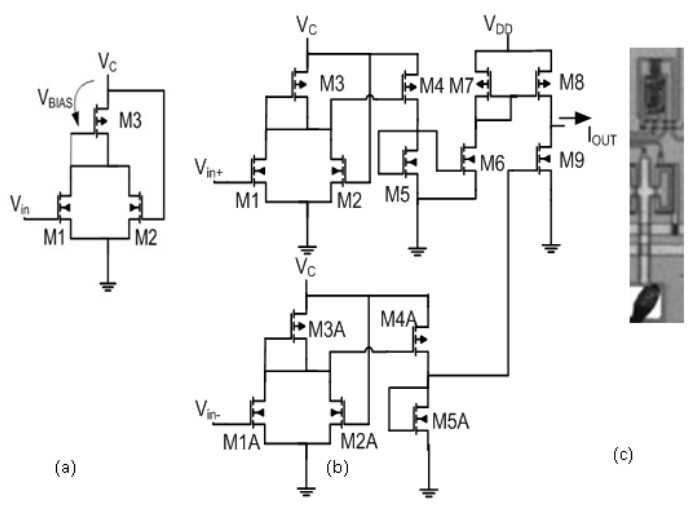

Figure 2. (a) Voltage-controlled linear conductance by a pair of MOSFETs in the triode region, (b) the tunable linear transconducance circuit, (c) the chip photograph of CMOS transconductor

A circuit of Figure 2 (a) was proposed as a voltagecontrolled linear conductance circuit by a PMOS transistor and a pair of identical NMOS transistors M1 and M2, while the conductance of MOS transistors is one of essential components in the analogue circuit design. The circuit of Figure 2 has been investigated for various neural networks applications, from implementing synapses to neuron $[4,5]$.

The total drain current $\mathrm{I}_{\mathrm{D}}$ of M1 and M2 can be expressed as

$$
\mathrm{I}_{\mathrm{D}}=\alpha\left(\mathrm{V}_{\mathrm{GS}}-\mathrm{V}_{\mathrm{BIAS}}-2 \mathrm{~V}_{\mathrm{TH}}\right)\left(\mathrm{V}_{\mathrm{C}}-\mathrm{V}_{\mathrm{BIAS}}\right)(1)
$$

where $\mathrm{V}_{\mathrm{GS}}=\mathrm{V}_{\text {inDC }} \pm \Delta \mathrm{V}_{\text {in }}$ is the gate-source voltage of transistor $\mathrm{M} 1, \mathrm{~V}_{\mathrm{DS}}=\mathrm{V}_{\mathrm{C}}-\mathrm{V}_{\mathrm{BIAS}}$ is the drain-source voltage of transistors $M 1$ and $M 2$, and $V_{C}$ is the tuning voltage of transconductance.
As the repeated two circuit in Figure 2(b) operate in the same condition, the output current $\mathrm{I}_{\mathrm{OUT}}$ is

$\mathrm{I}_{\mathrm{OUT}}=G m \Delta \mathrm{V}_{\text {in }}(2)$

where $\mathrm{I}_{\mathrm{OUT}}=\mathrm{I}_{\mathrm{D} 3}-\mathrm{I}_{\mathrm{DA}}$. The transconductance circuit of Figure 2(b) can be used as a programmable conductance of neuron's ion-channel or a synaptic connection with pulse/spike inputs.

The tuning properties of visual orientation selectivity have been believed to play the key role for perception in visual cortex as shown in Figure 3. The, the tuned feature map (or connection) of Figure 4(a) has been introduced to mimic the behaviour of brain in Figure 3. The proposed bio-inspired processing of Figure 4(a) is applied to example cases for evaluating its feasibility, as animal or human usually has the reasonable robustness. In this paper, the application of multi-directional selectivity on the video information is investigated further for the face or pose detection in various limited environment

The tuning properties of orientation selectivity have been believed to play the key role for perception in visual cortex. As shown in Figure 3 (a), the tuning of specific neurons to the orientation of visual stimulus probably depends on the tuning features after passive or active learning for the earlier processing of natural image. The rule we assume is very simple as illustrated in Figure 3 (b), though some modifications are likely necessary for being more plausible to the natural system. Here, the tuned feature map (or connection) of 5 x 5 synaptic weights is based on the reference stimulus to match, with the minor adjustment depending on the output. The tuned feature map of vertical, with the synaptic connections to 24 neurons (visual sensor, equivalent to a pixel) and itself. The synaptic weights are in the ration of (1: -0.6: 0.1 for black : grey : white). The six types of input stimulus (50 x 50 pixels) are experimented with the feature map (as synaptic connections) and spiking neurons based on $\mathrm{H}-\mathrm{H}$ formalism.

The VLSI neuron is implemented by CMOS transconductance circuit of Figure 2 in $0.18 \mu \mathrm{m}$ CMOS technology. The spike burst output like Figure 3(a) is observed by SPICE simulation, mimicking the neuromorphic visual cortex for its biological spike dynamics,

The feasibility of bio-inspired neuromorphic system is demonstrated with its plausibility to primary simple cell function of visual cortex with the tuned feature characteristics of other orientations. 


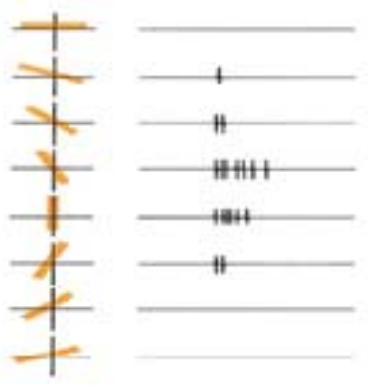

(a)

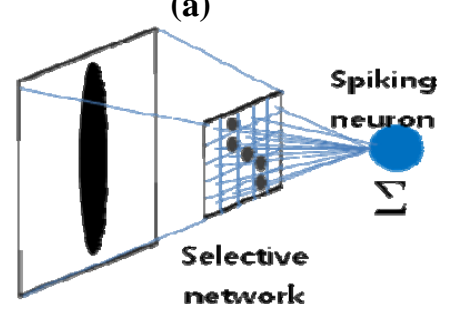

(b)

Figure 3. (a) Bio-inspired principles of user interface spiking pulse of neuron (b) Selective synapse networks

There are two major principles employed - first, the head of human has the high density if orientation components regardless of front view or side view. The other principle is the head linked to torso, while the arm is also linked to torso. Example cases are illustrated in Figure 4 to 6 , where the multidirectional orientation components are used prepare the orientation feature. The image of Figure 4 demonstrates face detection from the side-view, without frontal face features of eyes, nose or mouse. For the poor illumination condition and complicated environment, the proposed neuromorphic system is evaluated for the reflective lighting environment of Figure 5 and dark light condition of Figure 6.

The image of Figure 5 illustrates the complex orientation features, which are caused by both the poor illumination condition and complicated environment. The orientation features of test image shows the complicated non-human objects, such as the glossy pattern of marble floor, reflection on the mirror like floor, wall-mounted art work and etc. In fact, the outside wall of the building is the transparent glass which causes the continuous and substantial changes in the illumination level and direction. Those features from the environmental cause is reduced by taking the difference of extracted orientation features of two video frames, as the background of two video frames has the similar features. The noise characteristics in the shadow area on the floor still remain in the reference features by two video frames. The application of neural net head detector generates the neuromorphic visual image, which has the high lightened area of human head. The equivalent of action potential locates the human head in the image, based on the winner neuron. The head area is successfully detected, while the head detection is successful throughout the entire video from appearing to disappearing. During the video experimentation of human head detection, the pose detection is evaluated with the movements of right arm. The pose detection is motivated for the alternative user interface of TV like haptic input to smart phone, which allows the unconstrained natural environment. The additional process is to introduce another neural network detector to characterize the arm, torso and background. The prior head detection can determine the region of interest as the arm is attached to the torso, as the head is also attached to the torso.

The pose detection in Figure 5 is investigated for the new user experience interface of smart TV, which allows the unconstrained natural environment.
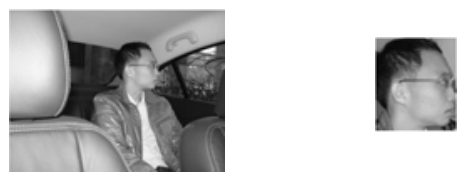

Figure 4. Human head detection with the side face

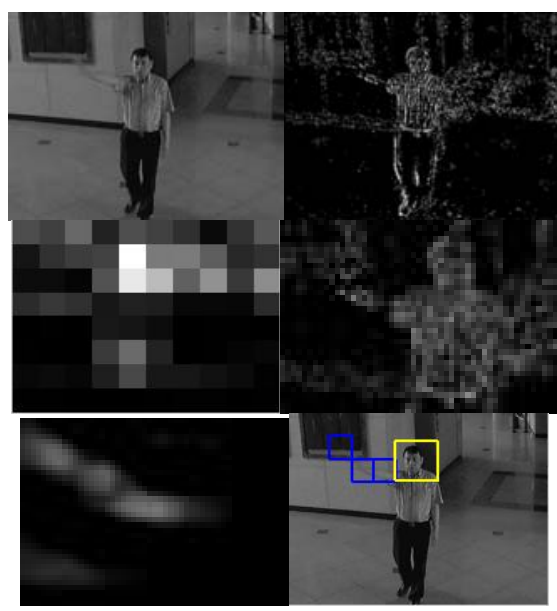

Figure 5. Detection of human pose based on the neuromorphic vision: top-left in clockwise, video image, difference of edge components from two frames, action level of detection neuron, region of interest for detecting the pose of arm, neural network arm pose detector, detected human head (yellow box) and arm pose (blue box)

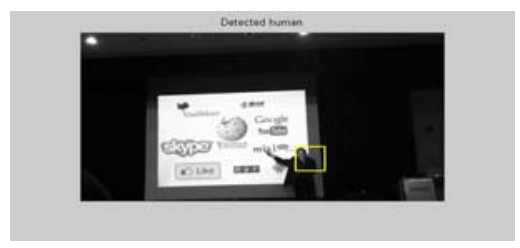

Figure 6. Head detection in the limited lighting 


\section{Conclusion}

The bio-inspired neuromorphic vision is proposed as a feasible way of implementing the user experience for smart IT service interface, with application examples of object detection of various human pose. The successful neuromorphic vision is explored for the system integration with neural networks for identifying the user interaction, based on neuromorphic processing with the neural network detector or histogram detector.

The successful detection in robust condition is demonstrated with the simple bio-inspired principles, and the neuromorphic vision is exhibited as enabling technology for applications in the natural user environment.

\section{Acknowledgement}

This research work was supported by LG U+ for Korea Communication Commission and NIPA, and also supported by (09 Transport System-Future 02) of Ministry of Land, Transport and Maritime, Korea

\section{References}

[1] F. Winkler et al, "Identity Management for IMS-based IPTV”, IEEE GlobeCom, IEEE, 2008, pp.1-5.

[2] J. Miller, “The User Experience (internet)", Internet Computing, IEEE, Sep 2005, pp. 90-92.

[3] S.J. Gibbs et al, "Evolving the TV Experience", Consumer Comm and Net Conference, IEEE, 2009, pp. 1-4.

[4] M. Risenhuber, and T. Poggio, " Hierarchical Models of Object Recognition in Cortex", Nature Neuroscience, 1999, pp. 1019-1025.

[5] M. Hausser, "The Hodgkin-Huxley Theory of Action Potential”, Nature Neuroscience Suppl, 2000, pp. 1165.

[6] W.J. Han, and I.S. Han, "Bio-inspired Visual Signal Processing Based on Neuromorphic Circuit", Proc. IMCAS , WSEAS, 2010, pp. 131-136 